\title{
For restorations short and long
}

Dental specialist BEGO is continuously expanding the extensive range of indications for its $3 \mathrm{D}$ printing system and is now offering a tooth-coloured composite for $3 \mathrm{D}$ printing of temporary crown and bridge constructions for short- and long-term restorations.

Next to CAD/Cast partial denture frames, surgical guides, dental models, individual impression trays and occlusal splints, dental technicians are now able to produce temporary crown and bridge restorations with the 3D printer Varseo $S$ and its precursors Varseo and Varseo L.

The wide range of indications of the new tooth-coloured composite in the colours A2, A3 and C2 according to the VITA classical colour system includes 3D printed crowns, inlays, onlays and veneers as well as bridges with a construction length of up to seven units and a pontic with maximum

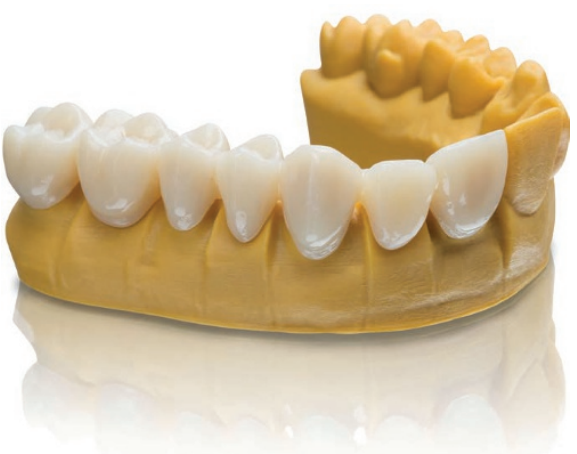

results at any time. The smooth surfaces of the printed objects allow a very good fit as well as a simple finishing. The printed restorations can be polished easily to a high gloss with standard composite polishers. Time-consuming post-processing is not required. Customisation of the objects for aesthetic characterisation is possible by means of light-curing stains.

The BEGO 3D printing system includes a diverse and scientifically proven range of materials, the Varseo S 3D printer, a light-curing device also as well as software

one molar width. Restorations made of VarseoSmile Temp are suitable for temporary use in the anterior and posterior region as short- and long-term restorations.

The specially developed printing and processing parameters ensure smooth production sequences with reproducible tools and a comprehensive service package. It was awarded the 'Best Of' seal of the INDUSTRPREIS in 2017 for the third consecutive year.

More information on the BEGO 3D printing system can be found at http:// www.bego.com/3d-printing/.

\section{The class-leading, speed-increasing handpiece}

The B.A. International German made speed increasing handpiece range allows unparalleled tactile sense, total speed adjustment and all the torque available.

An all-titanium body and uniquely ergonomic handpiece shape allows for a relaxed working style, whilst allowing for full visibility through built in fibre-optics.

High technology DLC coated bearings appear to be a small detail, but in fact allow for unparalleled smooth operation of this high precision instrument.

The FG chuck allows this speed increasing handpiece to use diamond or tungsten carbide turbine FG burs, which means that the discerning user can use their most appropriate and favourite burs, while always maintaining a stable electric motor working speed.

Quadruple spray guarantees perfect cooling at all times.

So confident is B.A. of its premium range of contra-angles that three years' manufacturer warranty is included in the price.

These premium state-of-the-art handpieces are of course also available at $1.45 \%$ APR on the unique and all-encompassing B.A. Total Care Subscription service.

For more information contact B.A. International on 0160477770 or its distributors Henry Schein Dental (01604 777700) or Kent Express (0800 0281181).

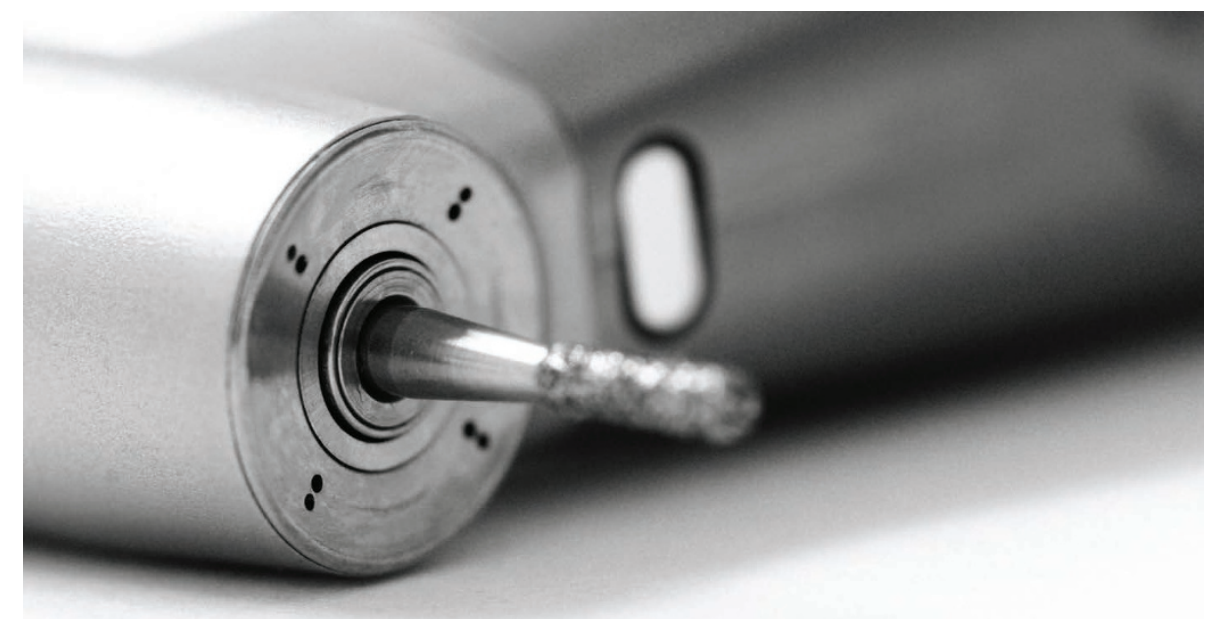

\section{Confidential professional advice for dentists}

Do you know a colleague who is struggling with mental health issues, problems with alcohol, drugs or an eating disorder? The Dentists' Health Support Trust can help. For confidential professional treatment and advice, call 02072244671 or visit www.dentistshealthsupporttrust.org.

The Dentists' Health Support Trust can provide:

- Advice and counselling from a mental health professional

- Treatment programme individually designed

- Inpatient treatment when appropriate

- Support and advice for friends, family and colleagues

- Assistance to return to work and support during recovery.

The Dentists'

Health Support Trust is a charity and entirely supported by donations. It is run by dentists for dentists. Charity number: 1003819. 